\title{
NEUROPSYCHOPHARMACOLOGY REVIEWS Integrating sleep, neuroimaging, and computational approaches for precision psychiatry
}

\author{
Andrea N. Goldstein-Piekarski ${ }^{1,2}$, Bailey Holt-Gosselin ${ }^{1}$, Kathleen $\mathrm{O}^{\prime}$ Hora $^{2}$ and Leanne M. Williams (iD) ${ }^{1,2}$
}

In advancing precision psychiatry, we focus on what imaging technology and computational approaches offer for the future of diagnostic subtyping and personalized tailoring of interventions for sleep impairment in mood and anxiety disorders. Current diagnostic criteria for mood and anxiety tend to lump different forms of sleep disturbance together. Parsing the biological features of sleep impairment and brain circuit dysfunction is one approach to identifying subtypes within these disorders that are mechanistically coherent and offer targets for intervention. We focus on two large-scale neural circuits implicated in sleep impairment and in mood and anxiety disorders: the default mode network and negative affective network. Through a synthesis of existing knowledge about these networks, we pose a testable framework for understanding how hyper- versus hypo-engagement of these networks may underlie distinct features of mood and sleep impairment. Within this framework we consider whether poor sleep quality may have an explanatory role in previously observed associations between network dysfunction and mood symptoms. We expand this framework to future directions including the potential for connecting circuit-defined subtypes to more distal features derived from digital phenotyping and wearable technologies, and how new discovery may be advanced through machine learning approaches.

Neuropsychopharmacology (2020) 45:192-204; https://doi.org/10.1038/s41386-019-0483-8

\section{INTRODUCTION}

Conceptualizing precision psychiatry

We are witnessing the emergence of precision medicine for psychiatry, that is, "Precision Psychiatry." We conceptualize precision psychiatry as an integrative approach that pulls together the scientific foundation of psychiatry with recent technological and computational advances, and directs them at closing the gap between discovery and clinical translation. However, "precision psychiatry" is a very broad term. In this review, we focus on precision approaches informed by neural circuits, quantified using neuroimaging technologies and computational models. We also touch on rapidly emerging digital phenotyping approaches that offer a means to move neural circuit insights into the field. This conceptualization of precision psychiatry is deployed in addressing at least three goals: explaining the heterogeneity of experienced disease in terms of coherent underlying neurobiological dimensions, using this mechanistic understanding to tailor interventions in a more personalized manner, and developing strategies for early detection and ultimately prevention. In this review we center on sleep impairment, a common hallmark of depression and anxiety disorders [1-6].

These inter-related objectives have been, respectively, considered in relation to the following precision approaches to other complex diseases: stratified medicine, personalized medicine, and precision health [7-9]. Stratified medicine focuses on identifying subgroups of patients who will benefit from treatments as a step toward a fully personalized approach that tailors treatments to individual people. Personalized medicine has focused on harnessing advances in biological markers (to date, primarily genomics) to select treatment options with the greatest likelihood of success. Precision health is the expansion of precision medicine to a wider concept of early detection and prevention that goes beyond a focus on disease.

There is an urgent need for precision medicine in general, and precision psychiatry in particular. Its emergence is part of the national focus on precision medicine [10] and precision health. In 2015, the Obama Administration launched the Precision Medicine Initiative, a major research effort aimed at improving health and changing the way we treat disease. In this initiative, precision medicine is described as "an innovative approach that takes into account individual differences in people's genes, environments, and lifestyles," thereby enabling doctors to tailor treatment to individual patients [11].

Why sleep, mood, and anxiety? Sleep, mood, and anxiety disorders are prevalent, costly, and commonly comorbid. Each year, an estimated 16 and 40 million American adults experience major depressive disorder (MDD) and anxiety disorders, respectively [12-14]. They are the leading causes of disability and lost productivity in the United States [15], estimated at over US\$200 billion per year [16]. They also commonly co-occur with sleep disruption, which results in more severe emotional distress than does either disorder alone $[17,18]$. Indeed, sleep disturbance is a core feature of both depression and anxiety [1-6]. For example, in a large sample of adults, $83 \%$ with depression and $77 \%$ with anxiety endorsed at least one insomnia complaint [19]. Conservative estimates indicate that individuals with mood and anxiety disorders are twice as likely to develop sleep difficulties as

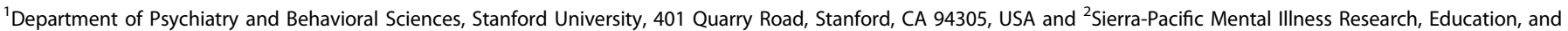
Clinical Center (MIRECC), Veterans Affairs Palo Alto Health Care System, 3801 Miranda Avenue, Palo Alto, CA 94304, USA

Correspondence: Leanne M. Williams (leawilliams@stanford.edu)

Received: 1 May 2019 Revised: 21 June 2019 Accepted: 22 July 2019

Published online: 19 August 2019 
those without $[4,19]$. Sleep complaints are often unresolved following treatment for both disorders and the residual sleep impairments are associated with significant risk for relapse [20]. Of particular concern, sleep disturbance is associated with an increased risk for suicide [21]. For example, insomnia and hypersomnia both confer a higher risk of suicide [20]. Critically, there is evidence that sleep disruption is a modifiable target that can reduce anxiety and depressive symptoms, making sleep targeted behavioral therapies attractive and tractable therapeutic targets [22-27]. Thus, optimizing the precision with which we can detect, parse, and intervene in this triad of disruptions has potentially sizable mental and public health benefits.

A neuroscience-informed approach to precision psychiatry. The federal Precision Medicine Initiative is paralleled by two federal research efforts focused specifically on psychiatry and neurosciences. First, the "BRAIN Initiative" aims to develop neurotechnologies for demystifying brain disorders, including psychiatric disorders [28]. This depth of understanding the brain will strengthen our ability to precisely identify dysfunction at the individual patient level. Second, the US National Institute of Mental Health is pioneering the Research Domain Criteria (RDoC) project [29], which has initiated a research approach to generating a neurobiologically valid framework for classifying psychiatric disorders and generating novel interventions related to neurobiological underpinnings.

In a neuroscience-informed approach to precision psychiatry, precise classification hinges on coherent mapping between neurobiological disruptions, symptoms, and behaviors, taking into account life experience and context [30]. Although the variance explained may be incremental, a key consideration is the identification of subtypes that help parse heterogeneity and are relevant to guiding treatment choices. In some instances, subtypes identified through a neuroscience-informed approach may align with the rich symptom classifications currently defined by the Diagnostic and Statistical Manual of Mental Disorders, 5th edition (DSM-5) [31]. In other cases, they may cut across diagnostic classifications [32] or reflect unforeseen, novel subtypes.

Applying a neuroscience-informed precision psychiatry approach to sleep, mood, and anxiety. In this review, we focus on the organ relevant to sleep impairment and its role in mood and anxiety disorders: the brain. In relation to the three goals of precision psychiatry outlined above, we review the current literature that characterizes impairments in sleep and how-through a more precise quantification of brain circuit dysfunction-we might parse these impairments in a more precise and neurobiologically valid manner. In doing so, we may identify particular subtypes of sleep impairment that characterize subtypes of mood and anxiety disorders, thus enabling us to better account for the heterogeneity of these disorders, develop mechanistically appropriate interventions, and detect early states of risk for developing these disorders. We focus on highly prevalent mood and anxiety disorders for which sleep disturbances are a diagnostic feature within the current DSM-5 criteria. Table 1 summarizes the diagnostic criteria for MDD and for generalized anxiety disorder. Highlighted are criteria 4 (change in sleep; insomnia or hypersomnia) and 6 (fatigue) for MDD, and criteria 2 (easily fatigued) and 6 (sleep disturbance-difficulty falling or staying asleep, or restless, unsatisfying sleep) within category $C$ for generalized anxiety disorder. We also focus on characterizing the overlap between large-scale neural circuits (or circuits) that have been implicated with sleep disruption and also show dysfunction in mood and anxiety disorders.

In order to set the scene for our review, we first provide an overview of relevant constructs from the sleep literature, and how we conceptualize these constructs within our precision psychiatry approach. We then organize the overview of imaging findings
Table 1. Diagnostic criteria for major depressive disorder and generalized anxiety disorder with sleep criteria indicated by yellow highlights

Major depressive disorder criteria

1 Depressed mood or irritable

2 Decreased interest or pleasure

3 Significant weight or appetite change

$4 \quad$ Change in sleep: Insomnia or hypersomnia

5 Change in activity

6 Fatigue or loss of energy

7 Guilt/worthlessness

8 Loss of concentration

9 Suicidality

Generalized anxiety disorder categories/criteria

A. Excessive anxiety and worry (apprehensive expectation)

B. The person finds it difficult to control the worry

C. A. and B. are accompanied with at least three of the following:

1. Edginess or restlessness

2. Tiring easily; more fatigued than usual

3. Impaired concentration or feeling as though the mind goes blank

4. Irritability

5. Increased muscle aches or soreness

6. Difficulty sleeping (due to trouble falling asleep or staying asleep, restlessness at night, or unsatisfying sleep)

with respect to sleep by parsing sleep disturbance into (1) poor quality or insufficient sleep and subjective tiredness, (2) insomnia (self-reported difficulty falling asleep or maintaining sleep), and (3) hypersomnia (we define as excessive tiredness despite receiving typically what is generally considered adequate sleep), as these are key aspects of the depression and anxiety diagnostic criteria that are typically grouped together.

Sleep brain-state associations with mood and anxiety. Sleep is separated into two main types: rapid eye movement (REM) sleep and non-REM (NREM) sleep, the latter being further subdivided into three stages (corresponding to increasing depth of sleep). These sleep stages are associated with dramatic alterations in functional brain activity and brain neurochemistry. For example, there is a substantial reduction in the levels of noradrenaline (norepinephrine) during REM sleep compared to noradrenaline levels during NREM sleep or wake [33-35]. In addition to epidemiological studies, studies using overnight electroencephalogram (EEG) sleep recordings of sleep (polysomnography (PSG)) also highlight how aspects of sleep architecture and physiology are associated with mood and anxiety features of emotional distress [36-41]. A lack of sleep continuity and depth is often accompanied by MDD and anxiety disorders [42]. A similar association is apparent for increased REM pressure [42]; however, some studies find the reverse for anxiety [43-46]. Spectral analysis of the EEG signal has shown that decreased delta activity during NREM is associated with greater perceptions of stress, and increased a-activity during NREM is associated with increased symptoms of depression [47]. Relatedly, individual differences in REM sleep spectral profiles are associated with next-day emotional function as assessed by brain function and behavioral emotion tasks $[36,37,39]$. In one such study, lower REM EEG $\gamma$-activity over prefrontal electrode sites was associated with a greater reduction of both amygdala and self-reported reactivity to emotional images [36]. Thus, we argue that in addition to utilizing functional magnetic resonance imaging (fMRI), EEG and similar sleep 
recording technologies that directly measure the brain are a promising brain target to be considered in our precision psychiatry framework.

Considering neural circuits as an explanatory framework for sleep, mood, and anxiety associations. We have chosen to limit the scope of our review to the default mode network (DMN) and the negative affective network (which includes core nodes of the salience network) due to their proposed role in governing reactivity and regulation of negative affect and mood (for a summary the main findings discussed below see Table 2). The literature search was conducted using the PubMed search engine in Papers version 3.4.20 with the following specifications: Sleep terms-sleep, insomnia, or hypersomnia; Imaging terms-fMRl; Network terms-default mode network, salience network, affective, negative emotion, or threat. See Supplementary Tables S1 and S2 for a more complete description of the study characteristics and findings of each study identified.

Default mode network

The default mode circuit (more typically known as the "DMN") is defined by the anterior medial prefrontal cortex (PFC), posterior cingulate cortex, and angular gyrus $[48,49]$ (Table 2$)$. This circuit is observed when the brain is probed under task-free conditions, and typically when participants are instructed to reflect on their own spontaneously generated thoughts $[50,51]$ (Table 2). Independent components analysis (ICA) suggests that the anterior and posterior regions define sub-networks of the DMN (for a review see [52]). This circuit also has a basis in structural white matter connections between the same regions $[53,54]$. Evidence from twin samples indicates that the DMN is engaged even during "rest" periods that occur between task stimuli, and this circuit is genetically heritable [54]. Aiding in clinical translation, there is now a method to summarize the degree of DMN functioning in a single number. This method can be applied to an individual to assess the degree of divergence from published norms [55]. The "default mode" may be considered a circuit that cuts across multiple domains of RDoC, including the Negative Valence and the Arousal and Regulatory systems domains ${ }^{1}$.

DMN features of sleep regulation

Given the association of the DMN with the arousal and regulatory systems, it is perhaps not surprising that it is one of the most highly studied networks with respect to sleep and consciousness. Simultaneous EEG and fMRI recording have enabled characterizations of changes in DMN activity and connectivity as a function of level of consciousness and sleep stage. Several studies have demonstrated that connectivity within the DMN weakens as a function of NREM sleep depth [56-61]. These effects have been observed using seed-based analyses, ICA, dynamic functional connectivity, and graph analytic approaches [56-61]. In contrast, the transition to REM sleep-a stage in which the EEG parallels arousal levels of wakefulness-restores DMN integrity to degrees that parallel wakefulness. Similarly, connectivity between nodes of the DMN and other networks implicated in mood and anxiety disorders fluctuate as a function of sleep stage. For example, connections between the DMN and hippocampus are strongest during periods of wakefulness and early stages of sleep, but weakened during slow-wave sleep [62]. These examinations help to establish how time spent in specific sleep stages may act on DMN functioning during sleep and translate to next-day functional alterations, which may in turn impact mood.

${ }^{1}$ http://www.nimh.nih.gov/research-priorities/rdoc/arousal-andregulatory-systems-workshop-proceedings.shtml https://www.nimh. nih.gov/research-priorities/rdoc/constructs/negative-valence-systems. shtml
DMN features of sleep debt, fatigue, and tiredness. Sleep debt, fatigue, and tiredness are key aspects of diagnostic criteria for major depressive and anxiety disorders that are typically grouped together (Table 1). In addition to demonstrating fluctuations during sleep, profiles of DMN connectivity have been associated with sleep debt, fatigue, and tiredness, core features of anxiety and depression. Functional connectivity and cerebral blood flow-as measured by arterial spin labeling within the DMN-appears to be strongest in the early morning and declines with increasing time spent awake [63,64]. Across individuals, worse quality sleep-including objective and subjective reports-is associated with a further weakening of DMN connectivity under task-free conditions in adolescents [65] as well as adults $[66,67]$. Increased daytime sleepiness has also been associated with decreased task-free functional connectivity within the DMN [68]. In contrast to within-DMN connectivity, connectivity between the DMN and regions outside the DMNincluding nodes within the negative affective network and hippocampus-increase as a function of worse sleep quality $[66,69,70]$. These studies parallel experimental sleep deprivation and sleep restriction paradigms that demonstrate loss of DMN integrity-both in terms of decreased within-DMN network connectivity and greater between-network connectivity under task-free conditions-in the sleep-deprived versus sleeprested conditions [71-74]. Taken together, these findings suggest that loss of DMN integrity may be a state marker for insufficient or poor-quality sleep, and that DMN integrity may be restored by improving sleep quality. This is particularly relevant when considering that hypo-connectivity of the DMN and poor sleep quality are both relevant to mood and anxiety (as discussed further in the section on intervention).

$D M N$ features of insomnia. Regarding other sleep characteristics of depressive and anxiety disorders, features of insomnia are also typically grouped together within an umbrella category of insomnia or hypersomnia (Table 1). However, findings from imaging of the DMN suggest that characteristics of insomnia may arise from underlying neural circuit disturbances that are distinct from those associated with other aspects of sleep disturbance. In contrast to the relative decreases in DMN connectivity under task-free conditions seen in conditions of insufficient and poor sleep quality, the most compelling findings with respect to insomnia are those that demonstrate a relative lack of deactivation of the DMN when compared to healthy controls [75], hyper-reactivity of the DMN in response to sleeprelated stimuli [76], and hyper-connectivity of the DMN under task-free conditions [77]. It has been hypothesized that the lack of disengagement may be reflective of increased self-reflective thought, rumination, and worry that may keep individuals from falling asleep [75]. However, it should be noted that other studies have found decreased rather than increased DMN connectivity [78-80].

Paralleling functional changes, decreased structural connectivity (as measured by structural covariance of gray matter volume calculated from T1-weighted images), and cortical thinning in DMN nodes has also been observed in those with insomnia [81].

$D M N$ markers of hypersomnia. Here we define hypersomnia as tiredness that persists despite receiving typically adequate sleep duration. While the neuroimaging studies described above have focused on subjective tiredness, to our knowledge no study to date has examined differences in brain profiles related to tiredness in those receiving adequate versus insufficient (or poor quality) sleep. Thus, it is currently unknown whether the hypo-connected DMN profile described in the section above also extends to those who may require more sleep than average. We believe this absence of studies focused on hypersomnia and brain function represents a critical gap in the literature given that hypersomnia is 


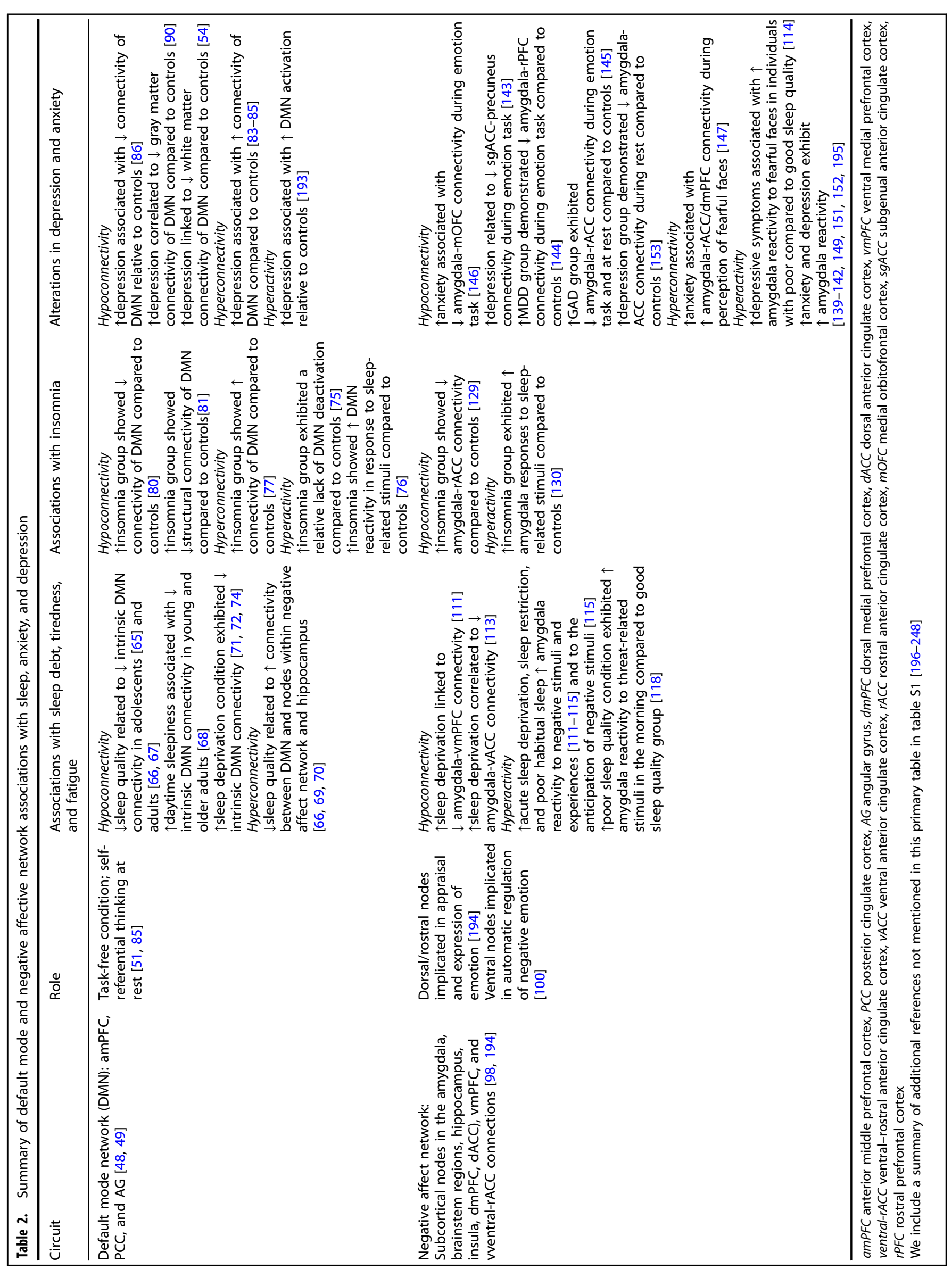


a feature of mood and anxiety disorders and is often associated with greater disability and poor outcomes $[2,82]$.

Could DMN dysfunctions in sleep parse mood and anxiety, and inform interventions accordingly?. Characterizing circuit dysfunctions that accompany sleep features may provide a new window into parsing risk factors for depression and anxiety, for subtyping based on circuits, and for informing intervention choices, as previously illustrated in a theoretical framework [30]. Mirroring the DMN profiles seen in insomnia, depression has been associated with over-activation and hyper-connectivity within the DMN relative to controls [83-85]. Similarly, DMN hyper-connectivity in MDD has been associated with higher levels of maladaptive rumination about depressive thoughts $[86,87]$, which may also contribute to difficulty falling asleep [75].

Intervention implications. Guided by insights in the basic sleep literature, poor sleep quality is unlikely to cause the DMN hyperengagement commonly seen in insomnia and depression given that poor sleep quality and insufficient sleep independently and robustly weaken rather than strengthen DMN connectivity [56-61]. Instead, it is plausible that the DMN hyper-engagement may induce both the insomnia and depressive mood features $[69,75]$, and may itself be a target of treatment [88, 89] (Fig. 1a). Indeed, interventions that decrease DMN connectivity may be particularly effective at improving insomnia and mood features.

For example, repetitive transcranial magnetic stimulation (rTMS) has been shown to be effective for reducing depressive symptoms [88] and improving sleep [89] while decreasing DMN connectivity. Sleep deprivation, which also decreases DMN connectivity, has been shown to improve mood in some individuals, while sleep restriction (a form of sleep deprivation) is a core component of cognitive behavioral therapy for insomnia [76] (Fig. 1b). However, it is still unknown whether individuals who have the highest degree of DMN over-engagement would benefit most from these treatments, or whether those who have the greatest decreases in DMN connectivity due to treatment receive the greatest reduction in mood and insomnia symptoms.
There is also evidence for DMN hypo-connectivity in MDD relative to controls [86], mirroring studies that reflect tiredness and situations of insufficient sleep. Alterations in brain structure also appear to be similar across conditions of sleep disruption and conditions seen in depression. MDD has been associated with decreased regional gray matter connectivity [90] and loss of white matter connectivity [91] within the DMN, particularly within the posterior sub-network. Widespread reductions in gray matter have also been observed across regions of the DMN and in nodes within interacting circuits [92].

Functional hypo-connectivity within the DMN, and between the DMN and other regions, in MDD has been associated with treatment resistance to pharmacotherapies [93-95]. In light of these findings, and in light of the association between DMN hypoconnectivity and poor sleep quality and sleep dept, individuals exhibiting DMN hypo-connectivity together with both poor sleep quality and depressive symptoms may benefit from targeted sleep therapies as an alternative or a lead in to antidepressant treatments (Fig. 1b). In contrast, functional hyper-connectivity within the DMN and with other regions as a promising marker of response to rTMS [95-97]. Thus, rTMS might be explored as an alternative intervention for sleep disturbances accompanying depression and anxiety, when DMN hyper-connectivity is the prominent neural circuit feature (Fig. 1b).

Negative affective network

Threat processing components of the negative affective circuits comprise the amygdala, hippocampus, insula, and dorsal and ventral portions of the PFC, including the dorsal medial PFC and its dorsal ACC connections, and the ventral medial PFC and its ventral (subgenual and pregenual)-rostral ACC connections [98, 99] (Table 1). The dorsal prefrontal sub-circuit has been preferentially implicated in appraisal and expression of emotion, and may be considered an "aversive amplification" sub-network [99] that serves to boost the processing of signals of potential threat [99]. Complementing this function, the ventral sub-circuit has been implicated in automatic regulation of negative emotion $[98,100]$ (for a review, see [101]; for meta-analysis, see [98]). These a

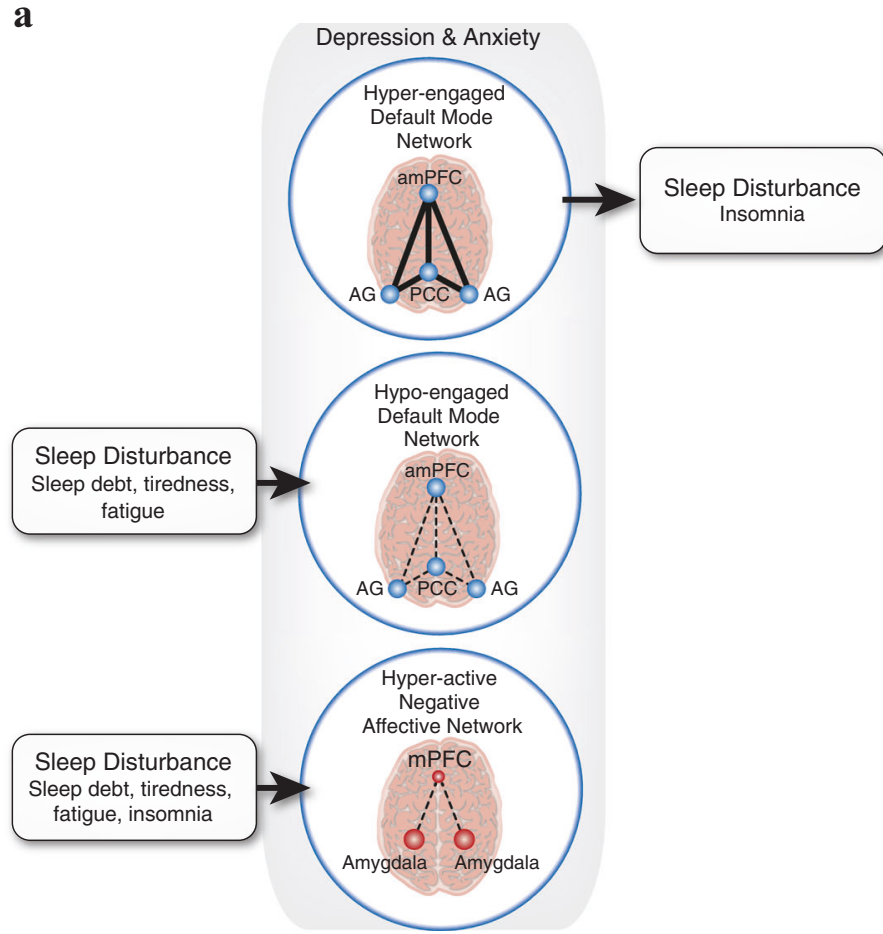

b
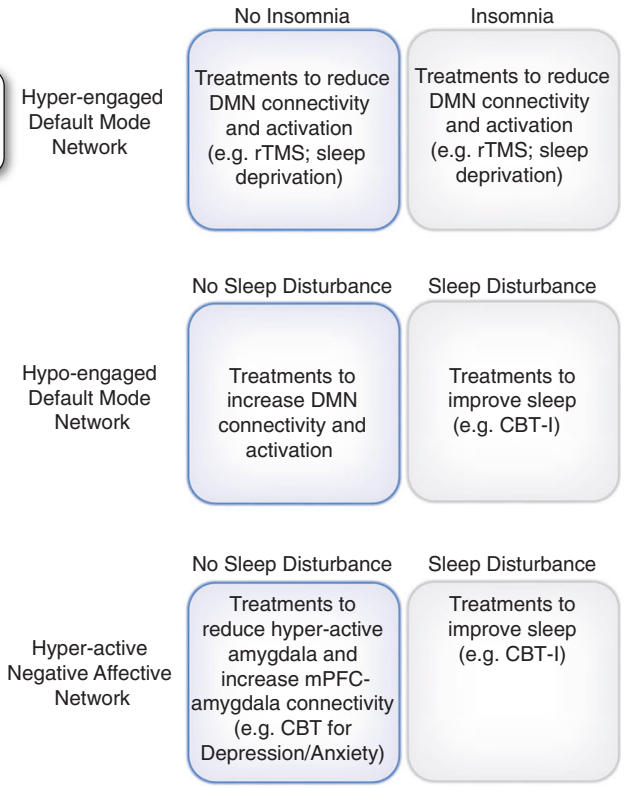

Fig. 1 a Hypothesized models accounting for sleep and network dysfunction in anxiety and depression and their respective $\mathbf{b}$ treatment implications 
components overlap with components of the salience circuit, and they may be engaged by the arousal and valence properties of threat stimuli, respectively.

These sub-networks may be engaged even in the absence of conscious sensory awareness [102] (for meta-analysis, see [98]). In light of their commonly observed co-activation [98], the negative affect circuit might subserve the perception of negative emotion cues and the salience circuit, the arousal aspects of feeling these emotions.

Direct activation of the amygdala and the prefrontal regions to which it projects may occur automatically even in the absence of explicit conscious evaluation [102-105] (for meta-analysis, see [98]). Similar bottom-up amygdala reactivity has been observed for masked presentations of other threat stimuli such as phobicrelevant cues [105].

Negative affective network features of sleep regulation. Due to the evoked nature of the negative affective network, relatively few studies have examined brain patterns of activity during sleep. However, early positron-emission tomography imaging studies identified increased glucose metabolism in regions belonging to the negative affective network-including the amygdala, ACC and medial PFC (mPFC)—during REM sleep [106]. More recent fMRI studies reveal similar increases in activity during REM sleep in negative emotion-related regions both subcortically (in the amygdala, striatum, and hippocampus) and cortically (in the insula and mPFC) [107-109].

Negative affective network features of sleep debt, fatigue, and tiredness. Several studies have demonstrated that the negative affective network is impacted by sleep status, particularly the amygdala and mPFC nodes. Acute sleep deprivation, sleep restriction, and poor habitual sleep all amplify amygdala reactivity to negative stimuli and experiences [110-115], and to the anticipation of negative stimuli [115]. Moreover, this signature of excessive amygdala reactivity caused by sleep disruption has routinely been linked to decreases in functional connectivity (both during tasks and at rest) within the mPFC [110-114].

These sleep-loss induced alterations in brain function are paired with changes in affective behavioral outcomes and subjective reports of significant clinical relevance. For example, inter-individual differences in mPFC-amygdala connectivity following sleep deprivation are significantly correlated with concurrent sleep-loss increases in subjective anxiety [113]. Moreover, sleep quality appears to moderate the relationships between amygdala reactivity and clinical symptoms. Greater amygdala reactivity to fearful faces was associated with greater depressive symptoms and perceived stress in those with poor compared to good sleep quality [114]. Poor sleep quality and insufficient sleep has also been associated with diminished ability to discriminate threatening facial expressions from neutral expressions [37], and with diminished emotion regulation brain function in the MPFC $[116,117]$. Critically, these same regions are impaired both by experimentally-induced and naturalistic sleep disruption, and may be improved by intervening sleep [36]. The impacts of prior sleep status on the negative affective network may also interact with circadian time-of-day effects: amygdala reactivity to threat-related stimuli was greater in the morning only for those with poor sleep quality, while those with good sleep quality showed little circadian modulation of amygdala reactivity [118].

With respect to structural findings, gray matter volume in negative affective network nodes of the MPFC and insula has been associated with inter-individual differences in sleep, including homeostatic-related NREM sleep oscillations $[119,120]$, daytime sleepiness [121], and the frequency of early morning awakenings [122]. In sum, the negative affective network appears to be particularly sensitive to poor sleep, demonstrating a relative hyper-reactive state together with loss of mPFC top-down control.

Negative affective network features of insomnia. Differences in amygdala, insula, and mPFC connectivity during task-free conditions have been observed in those with insomnia symptoms [77, 123-128]. One study, which explicitly focused on nodes within the negative affective network, found decreased amygdala-rostral ACC connectivity in those with insomnia relative to healthy controls [129]. These associations held when examining the linear relationships between objective and subjective sleep quality measures and amygdala-rostral ACC connectivity, with greater amygdala-rostral ACC connectivity being associated with better sleep quality. Very few studies have examined task-evoked negative affective network function in those with insomnia. Results of these studies have found increased amygdala responses to sleep-related stimuli and a decrease in amygdala habituation to the negative non-sleeprelated emotional images when compared to good sleepers [130]. However, it should be noted that many of these studies examined these associations in individuals without co-morbid anxiety and depression symptoms.

Negative affective network markers of hypersomnia. As was the case with the DMN, to the best of our knowledge no neuroimaging studies have focused on hypersomnia explicitly with respect to the negative affective network.

Could negative affect network dysfunctions in sleep parse mood and anxiety, and inform interventions accordingly?. There is a striking overlap between the pattern of negative affective network dysfunction, sleep disturbance, and anxiety and depression. Indeed, both hyper-reactivity of the amygdala and hypoconnectivity of mPFC-amygdala are theorized to contribute to the development and maintenance of emotional distress that underlies the maladaptive behaviors commonly observed in anxiety disorders and MDD, including increased negative bias [131-133], increased threat responsivity [134, 135], and poor emotional regulation [136-138]. Anxiety disorders and depression show similar profiles of dysfunction within the nodes of the negative affective network [30, 139-148], demonstrating hyper-reactivity in the amygdala [139-142, 149-152]), combined with reduced top-down control as measured by hypoconnectivity between the amygdala and MPFC during emotion tasks [143-147] and at rest [145, 153-155]. Moreover, the degree of reactivity and regulation in these nodes correlates with subjective experiences of emotional distress [156] as well as emotional behaviors [157].

Intervention implications. Sleep deprivation has been shown experimentally to induce increased negative mood states [158$162]$, as well as a negative affective network profile mimicking that of anxiety and depression [110, 111, 115] and improving sleep may normalize these brain changes [36]. Further, sleep disturbance is a strong predictive factor in anxiety and depressive disorders development [1-4] and prognosis [20, 163]. These findings suggest that at least for some individuals, maladaptive negative affective network function may be an intermediate step between sleep disruption and anxiety and mood features, and that sleep is a modifiable target through which emotional distress can be reduced (Fig. 1a). For individuals of this subtype, treating sleep issues would be necessary to alleviate both the maladaptive negative affective network brain function and the mood/anxiety features (Fig. 1b). This may explain the increased chance of anxiety and depression relapse in those for whom the sleep disturbances remain after treatment [20] and by targeting this subtype in particular may improve treatment effects of sleep interventions, which have already shown promise 
in reducing anxiety and depressive symptoms without considering negative affective network function [22-27].

Future research directions

The relatively siloed approach of studying brain function in sleep, mood, and anxiety separately has made it difficult to ascertain whether the brain dysfunction commonly observed in mood and anxiety disorders are an expression of the comorbidity of sleep disturbance or arise independently. This review marks a first step toward integrating knowledge from across fields. As a result of this approach, several interesting insights and testable hypotheses have emerged related to the DMN and Negative Affective Networks and their associations with insomnia and sleep quality. Beyond highlighting the need to more regularly integrate findings across domains, we also discuss several limitations that future work needs to address below.

Improved standardization of methods across studies. In both the sleep and neuroimaging fields, a lack of standardized common methodologies makes it difficult to fully interpret findings across individual studies. In our review, we found numerous studies that concentrated within the default node and negative affect networks using functional connectivity under task-free conditions; however, each study utilized a different approach (seed-based, ICA, graph approaches, ReHo, etc.) and targeted different regions within these networks (e.g., regions of both the frontal and parietal subnetworks of the default DMN have been used as seed regions for analyses [94, 164, 165]). Thus, each measure characterizes different aspects of connectivity, and each method subtlety (or sometimes not so subtlety) changes the interpretation of connectivity (we have reviewed the different resting-state methodology as well as their respective interpretations in [166]). Thus, it is hard to interpret whether the absence of an association in a connection found in one study that is present in another is a novel finding or a byproduct of the changing methods. For example, it is currently difficult to parse whether the apparent contradictory findings of both hyper- and hypo-connectivity of the DMN in MDD maybe a function of identifying different subgroups of depression (e.g., subtype that is resistant to pharmacotherapy), due to experimental factors such as choice of seed regions for analysis, or due to the characteristics of the sample (e.g., nonmedicated currently depressed versus medicated, adolescents versus older adults etc.). The same difficulty is true in the sleep field with the wide array of assays for subjective and objective sleep dysfunction (e.g., self-reported insomnia symptoms versus EEG based wake after sleep onset (WASO) versus EEG Delta power during slow wave sleep). These issues are compounded by the relatively small, non-diverse sample sizes in most studies, particularly in studies that use more advanced objective sleep measurements and neuroimaging.

Parsing state versus trait features with increased measurements. To our knowledge, we still know little about imaging and EEG sleep measurement in the context of randomized controlled trials or in naturalistic longitudinal assessments. We lack information about which features of brain function and sleep EEG signatures are stable, trait-like features that predispose individuals to developing mood features and which are reflective of an individual's current psychiatric symptom status. Moreover, to the best of our knowledge, no study to date has examined associations between changes in sleep and changes in DMN or Negative Affective Network function together following antidepressant or psychotherapy treatments.

Advances in sensing and wearable technologies for precision sleep measurement. To date, most sleep measurements in reported studies were conducted either through self-report or with a single night of recorded sleep using the current gold standard, PSG. Each method has its own weaknesses. While subjective self-report of sleep is valuable in its own right, particularly with respect to measuring the psychological distress in patients seeking treatment, these measures do not always correspond to objective measures of sleep $[167,168]$. This is particularly important when considering that short sleep duration measured by objective sleep measurements, but not self-report, has been associated with worse health outcomes and response to treatment (e.g., $[169,170])$. An overnight of PSG currently offers the greatest sensitivity for identifying sleep features related to brain function. However, a PSG recording requires highly trained lab technicians to adhere the electrodes, is relatively cumbersome for the participant, and is relatively expensive when done under lab settings. Taken together, these factors potentially limit the total number of subjects and make PSG recording difficult to conduct for multiple nights in a row. Rapid advances in technologyincluding smartphone apps that quantify rest activity by utilizing information about ambient light, screen usage, and the accelerometer from the phone itself, wearables ranging from more traditional wrist devices and other smartwatches [171, 172], to electrode based head bands [173, 174], smart beds [175], and noncontact sleep sensors [176-180] - open up new opportunities for long-term monitoring as well as characterizing new features of sleep and the stability of these brain features over time. However, it should be noted that these new devices are not without limitations. Few studies have been published validating these new technologies against more traditional sleep measurements, and in those cases, these devices tend to over- or under-estimate key sleep measurements such as total sleep time, sleep onset, and WASO [176, 178, 181, 182]. Nevertheless, these non-invasive home monitoring sleep measurement techniques still hold promise in characterizing sleep disturbances related to depression and anxiety. For example, students with higher depression severity, assessed by self-report on the PHQ-9, have significantly more sleep disturbance than students on average, as inferred from more variations in bed time (i.e., start time) and wake time (i.e., end time) [183] measured by passive smart-phone sampling. Similarly, sensor-derived metrics for sleep duration have also been associated with changes in depression assessed via the PHQ-9 [184]. Although these studies require cross-validation, replication, and tests of generalizability, they offer a preliminary validation of the utility of these measurements in relating to mood features in the wild.

Perhaps, most exciting when considering clinical application are the brain-based wearables capable of classifying sleep features in real time. These tools offer novel ways to intervene and potentially change physiological patterns of sleep that may be contributing to dysfunctional brain states and mood features during waking hours. For example, one study has already showed promise by utilizing a dry-EEG head band and real-time sleep quantification to detect and enhance slow wave sleep using closed-loop auditory stimulation in the participant's own bed, outside the sleep laboratory [185]. While the authors did not directly assess the impacts of increased slow wave activity on mood, this groundbreaking study provides an exciting proof of concept of a wearable delivered treatment that detects and modifies maladaptive sleep features tied to mood disorders in real-time every night at home (e.g., reduce REM density). Similarly, advances in machine learning algorithms offer new ways of identifying meaningful sleep features that characterize mood and anxiety states outside of the framework of traditional sleep staging, that are more tightly linked to biology and brain function. A similar approach has been used to approximate brain age from overnight sleep recordings [186].

Advances in sensing and wearable technologies for associating mood and anxiety with sleep features. Although a number of labs and companies are developing mobile technologies to assess and 
help people living with depression, to our knowledge these technologies have focused to date on depression as an umbrella category rather than on specific features such as sleep [28, 71]. Our contention is that a focus on specific features will ultimately be necessary for precision subtyping and for connecting wearable and sensor information to neural circuit markers.

As outlined above in regard to precision sleep technologies, physiological sensors offer a future means to connect peripheral objective markers of both sleep and depression/anxiety to neural circuit subtypes. Changes in physiological indices of heart rate and skin conductance data offer a means to monitor arousal fluctuations relevant to both mood states and to sleep. For example, heart rate variability has been consistently implicated in depression, anxiety, and in identification of those individuals who are not likely to respond to antidepressants [187-189]. Recent developments in technology demonstrate the viability of wearable patches with one to three electrodes for electrocardiogram recording of heart rate variability, and their relevance to depression and anxiety-related stress $[190,191]$. Although we do not know how such patch-recorded data may relate precisely to sleep and to neural circuits, the relevance of heart rate and related physiological indices to both sleep and to mood and anxiety disorders suggests that in the future relationships between these measures and with neural circuit function are worthy of investigation.

Another line of emerging enquiry is the study of multiple sensor data and the application of machine learning algorithms to determine which combination of sensors may be most useful for identifying features of depression, including sleep disturbance features. As one illustration of this approach, support vector machine approaches identifying a combination of accelerometry, skin temperature, calls, text messages, web use, and email use has been found to achieve classification accuracies of up to $90 \%$ for distinguishing the top versus bottom 20th percentile of participants in regard to self-reported sleep quality and perceived stress [192]. Of course, such findings require validation, replication, and more detailed investigation as to whether they apply only to the extremes of distributions of individuals and how they apply in clinical samples.

At the beginning of this review, in setting the scene for our approach to neuroscience-informed precision psychiatry, we highlighted the context of the RDoC initiative. Given that wearable and mobile sensing approaches for sleep, mood, and anxiety focusing in particular on physiological and mood features relevant to both the arousal and negative valence system domains of RDoC, we envision RDoC constructs as an important framework for future studies that tie together sensing modalities with neural circuits relevant to both domains, such as the DMN and negative affect circuits that are the focus of this review.

\section{SUMMARY CONCLUSION}

As highlighted throughout the review, rapidly emerging advances in precision quantification of human neural circuits, sleep technologies, and wearable sensing technologies offer a promising way forward for characterizing subtypes of mood and anxiety disorders for which sleep disturbances are a prominent feature. These advances offer a means to parse specific features of sleep disturbance that traditionally have been lumped together in classifying mood and anxiety disorders, and to develop precision markers for guiding intervention studies and for developing new interventions that target disrupted sleep mechanisms in these disorders.

\section{FUNDING AND DISCLOSURE}

LMW has received consultant fees from BlackThorn Therapeutics and scientific advisory board fees from Psyberguide of the One Mind Institute, for work unrelated to this review. LMW is supported by National Institutes of Health funding (R01 MH101496; U01 MH109985). ANG-P is supported by National Institutes of Health funding (UH2/UH3 HL132368). BH-G is supported by National Institutes of Health funding (U01 $\mathrm{MH} 109985)$. The authors declare no competing interests.

\section{ACKNOWLEDGEMENTS}

We acknowledge the editorial support of Jon Kilner, MS, MA (Pittsburgh, PA, USA).

\section{ADDITIONAL INFORMATION}

Supplementary Information accompanies this paper at (https://doi.org/10.1038/ s41386-019-0483-8).

Publisher's note: Springer Nature remains neutral with regard to jurisdictional claims in published maps and institutional affiliations.

\section{REFERENCES}

1. Ford DE, Kamerow DB. Epidemiologic study of sleep disturbances and psychiatric disorders. An opportunity for prevention? JAMA. 1989;262:1479-84.

2. Breslau N, Roth T, Rosenthal L, Andreski P. Sleep disturbance and psychiatric disorders: a longitudinal epidemiological study of young adults. Biol Psychiatry. 1996;39:411-8.

3. Morphy $H$, Dunn KM, Lewis M, Boardman HF, Croft PR. Epidemiology of insomnia: a longitudinal study in a UK population. Sleep. 2007;30:274-80.

4. Neckelmann D, Mykletun A, Dahl AA. Chronic insomnia as a risk factor for developing anxiety and depression. Sleep. 2007;30:873-80.

5. Harvey AG. Sleep and circadian functioning: critical mechanisms in the mood disorders? Annu Rev Clin Psychol. 2011;7:297-319.

6. Harvey AG, Murray G, Chandler RA, Soehner A. Sleep disturbance as transdiagnostic: consideration of neurobiological mechanisms. Clin Psychol Rev. 2011;31:225-35

7. Fernandes BS, Williams LM, Steiner J, Leboyer M, Carvalho A, Berk M. The new field of 'precision psychiatry'. BMC Med. 2017;15:80.

8. FORUM Academy of Medical Sciences. Stratified, personalised or P4 medicine: a new direction for placing the patient at the centre of healthcare and health education; 2015. https://acmedsci.ac.uk/viewFile/564091e072d41.pdf.

9. Personalized Medicine Coalition. The case for personalized medicine. 3rd ed.; 2014.

10. Hudson K, Lifton R, Patrick-Lake B, Burchard EG, Coles T, Collins R, et al. The Precision Medicine Initiative cohort program-Building a research foundation for 21 st century medicine. In: Precision Medicine Initiative (PMI) Working Group Report to the Advisory Committee to the Director, NIH, 2015. Available at: https://acd.od.nih.gov/documents/reports/DRAFT-PMI-WG-Report-9-11-2015508.pdf. Accessed October 7, 2018.

11. Office of the Press Secretary. Fact Sheet: President Obama's Precision Medicine Initiative; 2015. https:/obamawhitehouse.archives.gov/the-press-office/2015/ 01/30/fact-sheet-president-obama-s-precision-medicine-initiative.

12. Substance Abuse and Mental Health Services Administration. Results from the 2012 National Survey on Drug Use and Health: mental health findings. Rockville, MD: US Department Health and Human Services; 2013.

13. Somers JM, Goldner EM, Waraich P, Hsu L. Prevalence and incidence studies of anxiety disorders: a systematic review of the literature. Can J Psychiatry. 2006;51:100-13.

14. Weissman MM, Bland RC, Canino GJ, Faravelli C, Greenwald S, Hwu HG, et al. The cross-national epidemiology of panic disorder. Arch Gen Psychiatry. 1997;54:305-9.

15. Whiteford HA, Degenhardt L, Rehm J, Baxter AJ, Ferrari AJ, Erskine HE, et al. Global burden of disease attributable to mental and substance use disorders: findings from the Global Burden of Disease Study 2010. Lancet. 2013;382:1575-86

16. Roehrig C. Mental disorders top the list of the most costly conditions in the United States: \$201 billion. Health Aff (Millwood). 2016;35:1130-5.

17. Papadimitriou GN, Linkowski P. Sleep disturbance in anxiety disorders. Int Rev Psychiatry. 2005;17:229-36.

18. Wetherell JL, Le Roux H, Gatz M. DSM-IV criteria for generalized anxiety disorder in older adults: distinguishing the worried from the well. Psychol Aging. 2003;18:622-7.

19. Stewart R, Besset A, Bebbington P, Brugha T, Lindesay J, Jenkins R, et al. Insomnia comorbidity and impact and hypnotic use by age group in a national survey population aged 16 to 74 years. Sleep. 2006;29:1391-7. 
20. Nutt $D$, Wilson S, Paterson L. Sleep disorders as core symptoms of depression. Dialog- Clin Neurosci. 2008;10:329-36.

21. Sabo E, Reynolds CF 3rd, Kupfer DJ, Berman SR. Sleep, depression, and suicide. Psychiatry Res. 1991;36:265-77.

22. Manber R, Buysse DJ, Edinger J, Krystal A, Luther JF, Wisniewski SR, et al. Efficacy of cognitive-behavioral therapy for insomnia combined with antidepressant pharmacotherapy in patients with comorbid depression and insomnia: a randomized controlled trial. J Clin Psychiatry. 2016;77:e1316-e1323.

23. Freeman D, Sheaves B, Goodwin GM, Yu LM, Nickless A, Harrison PJ, et al. The effects of improving sleep on mental health (OASIS): a randomised controlled trial with mediation analysis. Lancet Psychiatry. 2017;4:749-58.

24. Belanger L, Harvey AG, Fortier-Brochu É, Beaulieu-Bonneau S, Eidelman P, et al. Impact of comorbid anxiety and depressive disorders on treatment response to cognitive behavior therapy for insomnia. J Consult Clin Psychol. 2016;84:659-67.

25. Luik Al, Bostock S, Chisnall L, Kyle SD, Lidbetter N, Baldwin N, et al. Treating depression and anxiety with digital cognitive behavioural therapy for insomnia: a real world NHS evaluation using standardized outcome measures. Behav Cogn Psychother. 2017;45:91-6.

26. Smith MT, Huang Ml, Manber R. Cognitive behavior therapy for chronic insomnia occurring within the context of medical and psychiatric disorders. Clin Psychol Rev. 2005;25:559-92.

27. Davidson JR, Dawson S, Krsmanovic A. Effectiveness of group Cognitive Behavioral Therapy For Insomnia (CBT-I) in a primary care setting. Behav Sleep Med. 2017;17:1-13.

28. Markoff J Obama seeking to boost study of human brain. New York Times. 2013.

29. Insel T, Cuthbert B, Garvey M, Heinssen R, Pine DS, Quinn K, et al. Research domain criteria (RDoC): toward a new classification framework for research on mental disorders. Am J Psychiatry. 2010;167:748-51.

30. Williams LM. Defining biotypes for depression and anxiety based on large-scale circuit dysfunction: a theoretical review of the evidence and future directions for clinical translation. Depress Anxiety. 2016;34:9-24.

31. American Psychiatric Association. Diagnostic and statistical manual of mental disorders: DSM-5. 5th ed. Washington, DC: American Psychiatric Association; 2013. p. 947. xliv

32. Grisanzio KA, Goldstein-Piekarski AN, Wang MY, Rashed Ahmed AP, Samara Z, Williams $L M$, et al. Transdiagnostic symptom clusters and associations with brain, behavior, and daily function in mood, anxiety, and trauma disorders. JAMA Psychiatry. 2018;75:201-9.

33. Shouse MN, Staba RJ, Saquib SF, Farber PR. Monoamines and sleep: microdialysis findings in pons and amygdala. Brain Res. 2000;860:181-9.

34. Park SP. In vivo microdialysis measures of extracellular norepinephrine in the rat amygdala during sleep-wakefulness. J Korean Med Sci. 2002;17:395-9.

35. Ouyang M, Hellman K, Abel T, Thomas SA. Adrenergic signaling plays a critical role in the maintenance of waking and in the regulation of REM sleep. J Neurophysiol. 2004;92:2071-82.

36. van der Helm E, Yao J, Dutt S, Rao V, Saletin JM, Walker MP. REM sleep depotentiates amygdala activity to previous emotional experiences. Curr Biol. 2011:21:2029-32.

37. Goldstein-Piekarski AN, Greer SM, Saletin JM, Walker MP. Sleep deprivation impairs the human central and peripheral nervous system discrimination of social threat. J Neurosci. 2015;35:10135-45.

38. Gujar N, McDonald SA, Nishida M, Walker MP. A role for REM sleep in recalibrating the sensitivity of the human brain to specific emotions. Cereb Cortex. 2010;21:115-23

39. Nishida Pearsal IJ, Buckner RL, Walker MP. REM sleep, prefrontal theta, and the consolidation of human emotional memory. Cereb Cortex. 2009;19:1158-66.

40. Mander BA, Santhanam S, Saletin JM, Walker MP. Wake deterioration and sleep restoration of human learning. Curr Biol. 2011;21:R183-4.

41. Saletin JM, Goldstein AN, Walker MP. The role of sleep in directed forgetting and remembering of human memories. Cereb Cortex. 2011;21:2534-41.

42. Baglioni C, Nanovska S, Regen W, Spiegelhalder K, Feige B, Nissen C. Sleep and mental disorders: meta-analysis of polysomnographic research. Psychol Bull. 2016;142:969-90.

43. Rapoport J, Elkins R, Langer DH, Sceery W, Buchsbaum MS, Gillin JC, et al. Childhood obsessive-compulsive disorder. Am J Psychiatry. 1981;138:1545-54.

44. Chevrette T, Bouvier H, Chevrier E, Godbout R. Sleep in adolescents with anxiety disorders. Sleep. 2005;28:A105.

45. Reynolds CF 3rd, Shaw DH, Newton TF, Coble PA, Kupfer DJ. EEG sleep in outpatients with generalized anxiety: a preliminary comparison with depressed outpatients. Psychiatry Res. 1983;8:81-9.

46. Monti JM, Monti D. Sleep disturbance in generalized anxiety disorder and its treatment. Sleep Med Rev. 2000;4:263-76.

47. Hall M, Buysse DJ, Nowell PD, Nofzinger EA, Houck P, Reynolds CFI, et al. Symptoms of stress and depression as correlates of sleep in primary insomnia. Psychosom Med. 2000;62:227-30.
48. Greicius MD, Krasnow B, Reiss AL, Menon V. Functional connectivity in the resting brain: a network analysis of the default mode hypothesis. Proc Natl Acad Sci USA. 2003;100:253-8.

49. Greicius MD, Supekar K, Menon V, Dougherty RF. Resting-state functional connectivity reflects structural connectivity in the default mode network. Cereb Cortex. 2009;19:72-8.

50. Seeley WW, Menon V, Schatzberg AF, Keller J, Glover GH, Kenna H, et al. Dissociable intrinsic connectivity networks for salience processing and executive control. J Neurosci. 2007;27:2349-56.

51. Shulman GL, Fiez JA, Corbetta M, Buckner RL, Miezin FM, Raichle $M E$, et al. Common blood flow changes across visual tasks: ii. decreases in cerebral cortex. J Cogn Neurosci. 1997;9:648-63.

52. Mulders PC, van Eijndhoven PF, Schene AH, Beckmann CF, Tendolkar I. Restingstate functional connectivity in major depressive disorder: a review. Neurosci Biobehav Rev. 2015:56:330-44.

53. Horn DI, Yu C, Steiner J, Buchmann J, Kaufmann J, Osoba A, et al. Glutamatergic and resting state functional connectivity correlates of severity in major depression-the role of pregenual anterior cingulate cortex and anterior insula. Front Syst Neurosci. 2010;4:1-10.

54. Korgaonkar MS, Ram K, Williams LM, Gatt JM, Grieve SM. Establishing the resting state default mode network derived from functional magnetic resonance imaging tasks as an endophenotype: a twins study. Hum Brain Mapp. 2014;35:3893-902.

55. Ball TM, Goldstein-Piekarski AN, Gatt JM, Williams LM. Quantifying person-level brain network functioning to facilitate clinical translation. Transl Psychiatry. 2017:7:e1248.

56. Chow HM, Horovitz SG, Carr WS, Picchioni D, Coddington N, Fukunaga M, et al. Rhythmic alternating patterns of brain activity distinguish rapid eye movement sleep from other states of consciousness. Proc Natl Acad Sci USA. 2013;110:10300-5.

57. Wu CW, Liu PY, Tsai PJ, Wu YC, Hung CS, Tsai YC, et al. Variations in connectivity in the sensorimotor and default-mode networks during the first nocturnal sleep cycle. Brain Connect. 2012;2:177-90.

58. Samann PG, Wehrle R, Hoehn D, Spoormaker VI, Peters H, Tully C, et al. Development of the brain's default mode network from wakefulness to slow wave sleep. Cereb Cortex. 2011;21:2082-93.

59. Larson-Prior LJ, Power JD, Vincent JL, Nolan TS, Coalson RS, Zempel J, et al. Modulation of the brain's functional network architecture in the transition from wake to sleep. Prog Brain Res. 2011;193:277-94.

60. Horovitz SG, Braun AR, Carr WS, Picchioni D, Balkin TJ, Fukunaga M, et al. Decoupling of the brain's default mode network during deep sleep. Proc Natl Acad Sci USA. 2009;106:11376-81.

61. Wilson RS, Mayhew SD, Rollings DT, Goldstone A, Przezdzik I, Arvanitis TN, et al. Influence of epoch length on measurement of dynamic functional connectivity in wakefulness and behavioural validation in sleep. Neuroimage. 2015;112:169-79.

62. Andrade KC, Spoormaker VI, Dresler M, Wehrle R, Holsboer F, Sämann PG, et al. Sleep spindles and hippocampal functional connectivity in human NREM sleep. J Neurosci. 2011;31:10331-9.

63. Hodkinson DJ, O'Daly O, Zunszain PA, Pariante CM, Lazurenko V, Zelaya FO, et al. Circadian and homeostatic modulation of functional connectivity and regional cerebral blood flow in humans under normal entrained conditions. J Cereb Blood Flow Metab. 2014;34:1493-9.

64. Blautzik J, Vetter C, Peres I, Gutyrchik E, Keeser D, Berman A, et al. Classifying fMRI-derived resting-state connectivity patterns according to their daily rhythmicity. Neuroimage. 2013;71:298-306.

65. Tashjian SM, Goldenberg D, Monti MM, Galván A. Sleep quality and adolescent default mode network connectivity. Soc Cogn Affect Neurosci. 2018;13:290-9.

66. Khalsa S, Mayhew SD, Przezdzik I, Wilson R, Hale J, Goldstone A, et al. Variability in cumulative habitual sleep duration predicts waking functional connectivity. Sleep. 2016;39:87-95.

67. Killgore WD, Schwab ZJ, Weiner MR. Self-reported nocturnal sleep duration is associated with next-day resting state functional connectivity. Neuroreport. 2012;23:741-5

68. Ward AM, McLaren DG, Schultz AP, Chhatwal J, Boot BP, Hedden T, et al. Daytime sleepiness is associated with decreased default mode network connectivity in both young and cognitively intact elderly subjects. Sleep. 2013;36:1609-15.

69. McKinnon AC, Hickie IB, Scott J, Duffy SL, Norrie L, Terpening Z, et al. Current sleep disturbance in older people with a lifetime history of depression is associated with increased connectivity in the default mode network. J Affect Disord. 2018:229:85-94.

70. Regen W, Kyle SD, Nissen C, Feige B, Baglioni C, Hennig J, et al. Objective sleep disturbances are associated with greater waking resting-state connectivity between the retrosplenial cortex/hippocampus and various nodes of the default mode network. J Psychiatry Neurosci. 2016;41:295-303. 
71. Ben Simon E, Maron-Katz A, Lahav N, Shamir R, Hendler T. Tired and misconnected: a breakdown of brain modularity following sleep deprivation. Hum Brain Mapp. 2017;38:3300-14.

72. Dai XJ, Liu CL, Zhou RL, Gong HH, Wu B, Gao L, et al. Long-term total sleep deprivation decreases the default spontaneous activity and connectivity pattern in healthy male subjects: a resting-state fMRI study. Neuropsychiatr Dis Treat. 2015;11:761-72.

73. Bosch OG, Rihm J, Scheidegger M, Landolt HS, Stämpfli P, Brakowski J, et al. Sleep deprivation increases dorsal nexus connectivity to the dorsolateral prefrontal cortex in humans. Proc Natl Acad Sci USA. 2013;110:19597-602.

74. Gujar N, Yoo SS, Hu P, Walker MP. The unrested resting brain: sleep deprivation alters activity within the default-mode network. J Cogn Neurosci. 2010;22:1637-48.

75. Marques DR, Gomes AA, Caetano G, Castelo-Branco M. Insomnia disorder and brain's default-mode network. Curr Neurol Neurosci Rep. 2018;18:45.

76. Kim SJ, Lee YJ, Kim N, Kim S, Choi JW, Park J, et al. Exploration of changes in the brain response to sleep-related pictures after cognitive-behavioral therapy for psychophysiological insomnia. Sci Rep. 2017;7:12528.

77. Ma X, Jiang G, Fu S, Fang J, Wu Y, Liu M, et al. Enhanced network efficiency of functional brain networks in primary insomnia patients. Front Psychiatry. 2018;9:46.

78. Nie X, Shao Y, Liu SY, Li HJ, Wan AL, Nie S, et al. Functional connectivity of paired default mode network subregions in primary insomnia. Neuropsychiatr Dis Treat. 2015;11:3085-93.

79. Dong $\mathrm{X}$, Qin H, Wu T, Hu H, Liao K, Cheng F, et al. Rest but busy: aberrant resting-state functional connectivity of triple network model in insomnia. Brain Behav. 2018;8:e00876.

80. Pang $R$, et al. Aberrant functional connectivity architecture in participants with chronic insomnia disorder accompanying cognitive dysfunction: a whole-brain, data-driven analysis. Front Neurosci. 2017;11:259.

81. Suh S, et al. Cortical thinning and altered cortico-cortical structural covariance of the default mode network in patients with persistent insomnia symptoms. Sleep. 2016;39:161-71.

82. Gooneratne NS, et al. Functional outcomes of excessive daytime sleepiness in older adults. J Am Geriatr Soc. 2003;51:642-9.

83. Kaiser $\mathrm{RH}$, et al. Large-scale network dysfunction in major depressive disorder: a meta-analysis of resting-state functional connectivity. JAMA Psychiatry. 2015;72:603-11.

84. Sheline $\mathrm{Yl}$, et al. Resting-state functional MRI in depression unmasks increased connectivity between networks via the dorsal nexus. Proc Natl Acad Sci USA. 2010;107:11020-5.

85. Greicius MD, et al. Resting-state functional connectivity in major depression: abnormally increased contributions from subgenual cingulate cortex and thalamus. Biol Psychiatry. 2007;62:429-37.

86. Zhu $X$, et al. Evidence of a dissociation pattern in resting-state default mode network connectivity in first-episode, treatment-naive major depression patients. Biol Psychiatry. 2012;71:611-7.

87. Hamilton JP, et al. Default-mode and task-positive network activity in major depressive disorder: implications for adaptive and maladaptive rumination. Biol Psychiatry. 2011;70:327-33.

88. George MS, et al. A controlled trial of daily left prefrontal cortex TMS for treating depression. Biol Psychiatry. 2000;48:962-70.

89. Diefenbach GJ, et al. Sleep improvements and associations with default mode network functional connectivity following rTMS for generalized anxiety disorder. Brain Stimul. 2019;12:184-86.

90. Singh MK, et al. Anomalous gray matter structural networks in major depressive disorder. Biol Psychiatry. 2013;74:777-85.

91. Korgaonkar, MS et al. Abnormal structural networks characterize major depressive disorder: a connectome analysis. Biol Psychiatry. 2014; 76:567-74.

92. Grieve SM, et al. Widespread reductions in gray matter volume in depression. Neuroimage Clin. 2013;3:332-9.

93. Guo WB, et al. Abnormal neural activity of brain regions in treatment-resistant and treatment-sensitive major depressive disorder: a resting-state fMRI study. J Psychiatr Res. 2012;46:1366-73.

94. Goldstein-Piekarski AN, et al. Intrinsic functional connectivity predicts remission on antidepressants: a randomized controlled trial to identify clinically applicable imaging biomarkers. Transl Psychiatry. 2018;8:57.

95. Dichter GS, Gibbs D, Smoski MJ. A systematic review of relations between resting-state functional-MRI and treatment response in major depressive disorder. J Affect Disord. 2015;172:8-17.

96. Ge R, et al. Abnormal functional connectivity within resting-state networks is related to $\mathrm{rTMS}$-based therapy effects of treatment resistant depression: a pilot study. J Affect Disord. 2017;218:75-81.
97. Liston $C$, et al. Default mode network mechanisms of transcranial magnetic stimulation in depression. Biol Psychiatry. 2014;76:517-26.

98. Kober $\mathrm{H}$, et al. Functional grouping and cortical-subcortical interactions in emotion: a meta-analysis of neuroimaging studies. Neuroimage. 2008;42:998-1031.

99. Robinson OJ, et al. The dorsal medial prefrontal (anterior cingulate) cortex-amygdala aversive amplification circuit in unmedicated generalised and social anxiety disorders: an observational study. Lancet Psychiatry. 2014;1:294-302.

100. Etkin $A$, et al. Failure of anterior cingulate activation and connectivity with the amygdala during implicit regulation of emotional processing in generalized anxiety disorder. Am J Psychiatry. 2010;167:545-54.

101. Phillips $M L$, et al. Neurobiology of emotion perception II: implications for major psychiatric disorders. Biol Psychiatry. 2003;54:515-28.

102. Williams LM, et al. Mode of functional connectivity in amygdala pathways dissociates level of awareness for signals of fear. J Neurosci. 2006;26:9264-71.

103. Liddell BJ, et al. A direct brainstem-amygdala-cortical 'alarm' system for subliminal signals of fear. Neuroimage. 2005;24:235-43.

104. Williams LM, et al. Amygdala-prefrontal dissociation of subliminal and supraliminal fear. Hum Brain Mapp. 2006;27:652-61.

105. Carlson JM, Reinke KS, Habib R. A left amygdala mediated network for rapid orienting to masked fearful faces. Neuropsychologia. 2009;47:1386-9.

106. Maquet $P$, et al. Functional neuroanatomy of human rapid-eye-movement sleep and dreaming. Nature. 1996;383:163-6.

107. Nofzinger EA. Functional neuroimaging of sleep. Semin Neurol. 2005;25:9-18.

108. Dang- $\mathrm{Vu} \Pi$, et al. Functional neuroimaging insights into the physiology of human sleep. Sleep. 2010;33:1589-603.

109. Miyauchi $S$, et al. Human brain activity time-locked to rapid eye movements during REM sleep. Exp Brain Res. 2009;192:657-67.

110. Yoo SS, et al. The human emotional brain without sleep-a prefrontal amygdala disconnect. Curr Biol. 2007;17:R877-8.

111. Chuah LY, et al. Sleep deprivation and interference by emotional distracters. Sleep. 2010;33:1305-13.

112. Killgore WD. Self-reported sleep correlates with prefrontal-amygdala functional connectivity and emotional functioning. Sleep. 2013;36:1597-608.

113. Motomura $Y$, et al. Sleep debt elicits negative emotional reaction through diminished amygdala-anterior cingulate functional connectivity. PLoS ONE. 2013;8:e56578.

114. Prather AA, Bogdan R, Hariri AR. Impact of sleep quality on amygdala reactivity, negative affect, and perceived stress. Psychosom Med. 2013;75:350-8.

115. Goldstein AN, et al. Tired and apprehensive: anxiety amplifies the impact of sleep loss on aversive brain anticipation. J Neurosci. 2013;33:10607-15.

116. Klumpp $H$, et al. Subjective and objective sleep quality modulate emotion regulatory brain function in anxiety and depression. Depress Anxiety. 2017;34:651-60.

117. Minkel JD, et al. Sleep quality and neural circuit function supporting emotion regulation. Biol Mood Anxiety Disord. 2012;2:22.

118. Baranger DAA, et al. An earlier time of scan is associated with greater threat-related amygdala reactivity. Soc Cogn Affect Neurosci. 2017; 12:1272-83.

119. Saletin JM, et al. Human hippocampal structure: a novel biomarker predicting mnemonic vulnerability to, and recovery from, sleep deprivation. J Neurosci. 2016;36:2355-63.

120. Saletin JM, van der Helm E, Walker MP. Structural brain correlates of human sleep oscillations. Neuroimage. 2013;83:658-68.

121. Killgore WD, et al. Voxel-based morphometric gray matter correlates of daytime sleepiness. Neurosci Lett. 2012;518:10-3.

122. Stoffers $D$, et al. Orbitofrontal gray matter relates to early morning awakening: a neural correlate of insomnia complaints? Front Neurol. 2012;3:105.

123. Zhang $X$, et al. Imbalanced spontaneous brain activity in orbitofrontal-insular circuits in individuals with cognitive vulnerability to depression. J Affect Disord. 2016;198:56-63.

124. Lu FM, et al. Disrupted topology of frontostriatal circuits is linked to the severity of insomnia. Front Neurosci. 2017;11:214.

125. Liu CH, et al. Reduced spontaneous neuronal activity in the insular cortex and thalamus in healthy adults with insomnia symptoms. Brain Res. 2016;1648 Part A:317-24.

126. Huang $Z$, et al. Abnormal amygdala connectivity in patients with primary insomnia: evidence from resting state fMRI. Eur J Radiol. 2012; 81:1288-95.

127. Yu S, et al. The imbalanced anterior and posterior default mode network in the primary insomnia. J Psychiatr Res. 2018;103:97-103.

128. Li C, et al. Aberrant effective connectivity of the right anterior insula in primary insomnia. Front Neurol. 2018;9:317 
129. Pace-Schott EF, et al. Resting state functional connectivity in primary insomnia, generalized anxiety disorder and controls. Psychiatry Res Neuroimaging. 2017;265:26-34.

130. Baglioni $C$, et al. Insomnia disorder is associated with increased amygdala reactivity to insomnia-related stimuli. Sleep. 2014;37:1907-17.

131. Habermas T, et al. Stuck in the past: negative bias, explanatory style, temporal order, and evaluative perspectives in life narratives of clinically depressed individuals. Depress Anxiety. 2008;25:E121-32.

132. Robinson OJ, et al. The role of serotonin in the neurocircuitry of negative affective bias: serotonergic modulation of the dorsal medial prefrontalamygdala 'aversive amplification' circuit. Neuroimage. 2013;78:217-23.

133. Dannlowski $U$, et al. Subliminal affective priming in clinical depression and comorbid anxiety: a longitudinal investigation. Psychiatry Res. 2006;143:63-75.

134. Yancey JR, Vaidyanathan U, Patrick CJ. Aversive startle potentiation and fear pathology: mediating role of threat sensitivity and moderating impact of depression. Int J Psychophysiol. 2015;98 Part 2:262-9.

135. Banks DM, Scott BG, Weems CF. Anxiety, hostile attributions, and differences in heart rate response to ambiguous situational vignettes in adolescents. Emotion. 2018;18:248-59.

136. Ehring $T$, et al. Emotion regulation and vulnerability to depression: spontaneous versus instructed use of emotion suppression and reappraisal. Emotion. 2010;10:563-72.

137. Mennin DS, et al. Preliminary evidence for an emotion dysregulation model of generalized anxiety disorder. Behav Res Ther. 2005;43:1281-310.

138. Gross JJ, Munoz RF. Emotion regulation and mental-health. Clin PsycholSci Pract. 1995;2:151-64.

139. Peluso MA, et al. Amygdala hyperactivation in untreated depressed individuals. Psychiatry Res. 2009;173:158-61.

140. Yang $\Pi$, et al. Adolescents with major depression demonstrate increased amygdala activation. J Am Acad Child Adolesc Psychiatry. 2010;49:42-51.

141. Killgore WD, et al. Cortico-limbic responses to masked affective faces across PTSD, panic disorder, and specific phobia. Depress Anxiety. 2014;31:150-9.

142. Bishop SJ, Duncan J, Lawrence AD. State anxiety modulation of the amygdala response to unattended threat-related stimuli. J Neurosci. 2004;24:10364-8.

143. Ho TC, et al. Functional connectivity of negative emotional processing in adolescent depression. J Affect Disord. 2014;155:65-74.

144. Kong $\mathrm{L}$, et al. Functional connectivity between the amygdala and prefrontal cortex in medication-naive individuals with major depressive disorder. J Psychiatry Neurosci. 2013;38:417-22.

145. Prater KE, et al. Aberrant amygdala-frontal cortex connectivity during perception of fearful faces and at rest in generalized social anxiety disorder. Depress Anxiety. 2013:30:234-41.

146. Hahn A, et al. Reduced resting-state functional connectivity between amygdala and orbitofrontal cortex in social anxiety disorder. Neuroimage. 2011;56:881-9.

147. Demenescu LR, et al. Amygdala activation and its functional connectivity during perception of emotional faces in social phobia and panic disorder. J Psychiatr Res. 2013;47:1024-31.

148. Williams LM. Precision psychiatry: a neural circuit taxonomy for depression and anxiety. Lancet Psychiatry. 2016;3:472-80.

149. Stein $M B$, et al. Increased amygdala and insula activation during emotion processing in anxiety-prone subjects. Am J Psychiatry. 2007;164:318-27.

150. Bryant RA, et al. Enhanced amygdala and medial prefrontal activation during nonconscious processing of fear in posttraumatic stress disorder: an fMRI study. Hum Brain Mapp. 2008;29:517-23.

151. Etkin A, et al. Individual differences in trait anxiety predict the response of the basolateral amygdala to unconsciously processed fearful faces. Neuron. 2004:44:1043-55.

152. Bryant RA, et al. Rostral anterior cingulate volume predicts treatment response to cognitive-behavioural therapy for posttraumatic stress disorder. J Psychiatry Neurosci. 2008:33:142-6.

153. Pannekoek JN, et al. Aberrant resting-state functional connectivity in limbic and salience networks in treatment-naive clinically depressed adolescents. J Child Psychol Psychiatry. 2014;55:1317-27.

154. Zhang $X$, et al. First-episode medication-naive major depressive disorder is associated with altered resting brain function in the affective network. PLoS ONE. 2014;9:e85241.

155. Etkin A, et al. Disrupted amygdalar subregion functional connectivity and evidence of a compensatory network in generalized anxiety disorder. Arch Gen Psychiatry. 2009;66:1361-72.

156. Somerville $\mathrm{LH}$, et al. Human amygdala responses during presentation of happy and neutral faces: correlations with state anxiety. Biol Psychiatry. 2004;55:897-903.

157. El Khoury-Malhame M, et al. Amygdala activity correlates with attentional bias in PTSD. Neuropsychologia. 2011;49:1969-73.

158. Williams LM, et al. The ENGAGE study: Integrating neuroimaging, virtual reality and smartphone sensing to understand self-regulation for managing depression and obesity in a precision medicine model. Behav Res Ther. 2018;101:58-70.

159. Sagaspe $P$, et al. Effects of sleep deprivation on color-word, emotional, and specific stroop interference and on self-reported anxiety. Brain Cogn. 2006;60:76-87.

160. Babson KA, et al. An experimental investigation of the effects of acute sleep deprivation on panic-relevant biological challenge responding. Behav Ther. 2009;40:239-50.

161. Babson KA, et al. A test of the effects of acute sleep deprivation on general and specific self-reported anxiety and depressive symptoms: an experimental extension. J Behav Ther Exp Psychiatry. 2010;41:297-303.

162. Dinges DF, et al. Cumulative sleepiness, mood disturbance, and psychomotor vigilance performance decrements during a week of sleep restricted to $4-5 \mathrm{~h}$ per night. Sleep. 1997;20:267-77.

163. Marcks BA, Weisberg RB, Edelen MO, Keller MB. The relationship between sleep disturbance and the course of anxiety disorders in primary care patients. Psychiatry Res. 2010; 178:487-92.

164. Connolly CG, et al. Resting-state functional connectivity of subgenual anterior cingulate cortex in depressed adolescents. Biol Psychiatry. 2013; 74:898-907.

165. Alexopoulos GS, et al. Functional connectivity in the cognitive control network and the default mode network in late-life depression. J Affect Disord. 2012;139:56-65.

166. Lv H, et al. Resting-state functional MRI: everything that nonexperts have always wanted to know. Am J Neuroradiol. 2018;39:1390-9.

167. Carskadon MA, et al. Self-reports versus sleep laboratory findings in 122 drugfree subjects with complaints of chronic insomnia. Am J Psychiatry. 1976;133:1382-8.

168. Frankel BL, et al. Recorded and reported sleep in chronic primary insomnia. Arch Gen Psychiatry. 1976;33:615-23.

169. Bathgate $\mathrm{CJ}$, et al. Objective but not subjective short sleep duration associated with increased risk for hypertension in individuals with insomnia. Sleep. 2016;39:1037-45.

170. Bathgate CJ, Edinger JD, Krystal AD. Insomnia patients with objective short sleep duration have a blunted response to cognitive behavioral therapy for insomnia. Sleep. 2017;40:1.

171. Montgomery-Downs $\mathrm{HE}$, Insana SP, Bond JA. Movement toward a novel activity monitoring device. Sleep Breath. 2012;16:913-7.

172. Lee J, Finkelstein J. Consumer sleep tracking devices: a critical review. Stud Health Technol Inf. 2015;210:458-60.

173. Shambroom JR, Fabregas SE, Johnstone J. Validation of an automated wireless system to monitor sleep in healthy adults. J Sleep Res. 2012;21:221-30.

174. Chambon S, et al. DOSED: a deep learning approach to detect multiple sleep micro-events in EEG signal. J Neurosci Methods. 2019:321:64-78.

175. Kim Y, et al. Evaluation of unconstrained monitoring technology used in the Smart Bed for u-health environment. Telemed J E Health. 2011;17:435-41.

176. Tuominen J, et al. Sleep parameter assessment accuracy of a consumer home sleep monitoring ballistocardiograph beddit sleep tracker: a validation study. J Clin Sleep Med. 2019;15:483-7.

177. Paalasmaa J, et al. Unobtrusive online monitoring of sleep at home. Conf Proc IEEE Eng Med Biol Soc. 2012;2012:3784-8.

178. Perez-Macias JM, et al. Comparative assessment of sleep quality estimates using home monitoring technology. Conf Proc IEEE Eng Med Biol Soc. 2014;2014:4979-82

179. Seeton R, Adler A. Sensitivity of a single coil electromagnetic sensor for noncontact monitoring of breathing. Conf Proc IEEE Eng Med Biol Soc. 2008;2008:518-21.

180. De Chazal $P$, et al. Sleep/wake measurement using a non-contact biomotion sensor. J Sleep Res. 2011;20:356-66.

181. de Zambotti $M$, et al. Evaluation of a consumer fitness-tracking device to assess sleep in adults. Chronobiol Int. 2015;32:1024-8.

182. Lorenz CP, Williams AJ. Sleep apps: what role do they play in clinical medicine?. Curr Opin Pulm Med. 2017;23:512-6.

183. Chen Z, Lin M, Chen F, Lane ND, Cardone G, Wang R, et al. Unobtrusive sleep monitoring using smartphones. In: Seventh International Conference on Pervasive Computing Technologies for Healthcare and Workshops. Venice, Italy: IEEE; 2013.

184. Ben-Zeev D, et al. Next-generation psychiatric assessment: using smartphone sensors to monitor behavior and mental health. Psychiatr Rehabil J. 2015;38:218-26.

185. Cho $\mathrm{CH}$, et al. Mood prediction of patients with mood disorders by machine learning using passive digital phenotypes based on the circadian rhythm: prospective observational cohort study. J Med Internet Res. 2019;21:e11029.

186. Sun $\mathrm{H}$, et al. Brain age from the electroencephalogram of sleep. Neurobiol Aging. 2019;74:112-20. 
187. Kemp $\mathrm{AH}$, et al. Impact of depression and antidepressant treatment on heart rate variability: a review and meta-analysis. Biol Psychiatry. 2010;67:1067-74.

188. Kemp AH, Quintana DS. The relationship between mental and physical health: insights from the study of heart rate variability. Int J Psychophysiol. 2013;89:288-96.

189. Kircanski K, Williams LM, Gotlib $\mathrm{IH}$. Heart rate variability as a biomarker of anxious depression response to antidepressant medication. Depress Anxiety. 2019;36:63-71.

190. Lee WK, Yoon H, Park KS. Smart ECG monitoring patch with built-in R-peak detection for long-term HRV analysis. Ann Biomed Eng. 2016;44:2292-301.

191. Weenk $M$, et al. Stress measurement in surgeons and residents using a smart patch. Am J Surg. 2018;216:361-8.

192. Sano A, et al. Recognizing academic performance, sleep quality, stress level, and mental health using personality traits, wearable sensors and mobile phones. In: International Conference of Wearable Implant Body Sensor Network; 2015. https://www.ncbi.nlm.nih.gov/pmc/articles/PMC5431072/.

193. Sheline $\mathrm{Yl}$, et al. The default mode network and self-referential processes in depression. Proc Natl Acad Sci USA. 2009;106:1942-7.

194. Robinson OJ, et al. Towards a mechanistic understanding of pathological anxiety: the dorsal medial prefrontal-amygdala 'aversive amplification' circuit in unmedicated generalized and social anxiety disorders. Lancet Psychiatry. 2014;1:294-302.

195. Tashjian SM, Goldenberg D, Galvan A. Neural connectivity moderates the association between sleep and impulsivity in adolescents. Dev Cogn Neurosci. 2017; $27: 35-44$

196. Nilsonne G, et al. Intrinsic brain connectivity after partial sleep deprivation in young and older adults: results from the Stockholm Sleepy Brain study. Sci Rep. 2017;7:9422.

197. Spoormaker Vl, et al. Ventromedial prefrontal cortex activity and rapid eye movement sleep are associated with subsequent fear expression in human subjects. Exp Brain Res. 2014;232:1547-54.

198. Sheng J, et al. High definition-transcranial direct current stimulation changes older adults' subjective sleep and corresponding resting-state functional connectivity. Int J Psychophysiol. 2018;129:1-8.

199. Tushaus L, et al. Resisting sleep pressure: impact on resting state functional network connectivity. Brain Topogr. 2017;30:757-73.

200. Zhou $X$, et al. Sleep deprivation makes the young brain resemble the elderly brain: a large-scale brain networks study. Brain Connect. 2017;7:58-68.

201. Wang $L$, et al. Sleep deprivation disturbed regional brain activity in healthy subjects: evidence from a functional magnetic resonance-imaging study. Neuropsychiatr Dis Treat. 2016;12:801-7.

202. Samann PG, et al. Increased sleep pressure reduces resting state functional connectivity. MAGMA. 2010;23:375-89.

203. Wang $Y$, et al. Module number of default mode network: inter-subject variability and effects of sleep deprivation. Brain Res. 2015;1596:69-78.

204. Zhao R, et al. Disrupted resting-state functional connectivity in hippocampal subregions after sleep deprivation. Neuroscience. 2019;398:37-54.

205. Wilson RS, et al. Objective and subjective measures of prior sleep-wake behavior predict functional connectivity in the default mode network during NREM sleep. Brain Behav. 2019;9:e01172.

206. Chen WH, et al. Dissociable effects of sleep deprivation on functional connectivity in the dorsal and ventral default mode networks. Sleep Med. 2018;50:137-44.

207. $\mathrm{Xu} \mathrm{H}$, et al. Impact of $36 \mathrm{~h}$ of total sleep deprivation on resting-state dynamic functional connectivity. Brain Res. 2018;1688:22-32.

208. Kong $D$, et al. Altered long- and short-range functional connectivity density in healthy subjects after sleep deprivations. Front Neurol. 2018;9:546.

209. Uehara T, et al. Efficiency of a "small-world" brain network depends on consciousness level: a resting-state FMRI study. Cereb Cortex. 2014;24:1529-39.

210. Watanabe T, et al. Network-dependent modulation of brain activity during sleep. Neuroimage. 2014;98:1-10.

211. De Havas JA, et al. Sleep deprivation reduces default mode network connectivity and anti-correlation during rest and task performance. Neuroimage. 2012;59:1745-51.

212. Koike $T$, et al. Connectivity pattern changes in default-mode network with deep non-REM and REM sleep. Neurosci Res. 2011;69:322-30.

213. Dang-Vu T, et al. Spontaneous neural activity during human slow wave sleep. Proc Natl Acad Sci USA. 2008;105:15160-5.

214. Horovitz SG, et al. Low frequency BOLD fluctuations during resting wakefulness and light sleep: a simultaneous EEG-fMRI study. Hum Brain Mapp. 2008;29:671-82.

215. Facer-Childs ER, et al. Circadian phenotype impacts the brain's resting state functional connectivity, attentional performance and sleepiness. Sleep. 2019;42:1-12.
216. Poudel GR, Innes $\mathrm{CRH}$, Jones RD. Temporal evolution of neural activity and connectivity during microsleeps when rested and following sleep restriction. Neuroimage. 2018;174:263-73.

217. Wang $\mathrm{L}$, et al. Altered default mode and sensorimotor network connectivity with striatal subregions in primary insomnia: a resting-state multi-band fMRI study. Front Neurosci. 2018;12:917.

218. Lee YG, et al. Changes in subcortical resting-state functional connectivity in patients with psychophysiological insomnia after cognitive-behavioral therapy: changes in resting-state FC after CBT for insomnia patients. Neuroimage Clin 2018;17:115-23.

219. Riemann D, et al. Chronic insomnia and MRI-measured hippocampal volumes: a pilot study. Sleep. 2007;30:955-8.

220. Wu Y, et al. Abnormal topology of the structural connectome in the limbic cortico-basal-ganglia circuit and default-mode network among primary insomnia patients. Front Neurosci. 2018;12:860.

221. Li Z, et al. Disrupted brain network topology in chronic insomnia disorder: a resting-state fMRI study. Neuroimage Clin. 2018;18:178-85.

222. Wallace ML, et al. Using optimal combined moderators to define heterogeneity in neural responses to randomized conditions: application to the effect of sleep loss on fear learning. Neuroimage. 2018;181:718-27.

223. Guadagni V, et al. Sleep quality and its association with the insular cortex in emotional empathy. Eur J Neurosci. 2018;48:2288-300.

224. Feng $P$, et al. Alter spontaneous activity in amygdala and vmPFC during fear consolidation following 24h sleep deprivation. Neuroimage. 2018:172:461-9.

225. Goldstein-Piekarski AN, et al. Sex, sleep deprivation, and the anxious brain. $J$ Cogn Neurosci. 2018;30:565-78.

226. Motomura $Y$, et al., Two days' sleep debt causes mood decline during resting state via diminished amygdala-prefrontal connectivity. Sleep. 2017;40:1-9.

227. Uy JP, Galvan A. Sleep duration moderates the association between insula activation and risky decisions under stress in adolescents and adults. Neuropsychologia. 2017;95:119-29.

228. Motomura $\mathrm{Y}$, et al. Recovery from unrecognized sleep loss accumulated in daily life improved mood regulation via prefrontal suppression of amygdala activity. Front Neurol. 2017;8:306

229. Schneider $M$, et al. Spontaneous pupil dilations during the resting state are associated with activation of the salience network. Neuroimage. 2016;139:189-201.

230. Reidy BL, et al. Decreased sleep duration is associated with increased fMR responses to emotional faces in children. Neuropsychologia. 2016;84:54-62.

231. Tempesta $D$, et al. Emotional memory processing is influenced by sleep quality. Sleep Med. 2015;16:862-70.

232. Sterpenich $V$, et al. Sleep sharpens sensory stimulus coding in human visual cortex after fear conditioning. Neuroimage. 2014;100:608-18.

233. Payne JD, Kensinger EA. Sleep leads to changes in the emotional memory trace: evidence from FMRI. J Cogn Neurosci. 2011;23:1285-97.

234. Rosales-Lagarde $A$, et al. Enhanced emotional reactivity after selective REM sleep deprivation in humans: an fMRI study. Front Behav Neurosci. 2012;6:25.

235. Heine L, et al. Resting state networks and consciousness: alterations of multiple resting state network connectivity in physiological, pharmacological, and pathological consciousness states. Front Psychol. 2012;3:295.

236. Vallat $R$, et al. Hard to wake up? The cerebral correlates of sleep inertia assessed using combined behavioral, EEG and fMRI measures. Neuroimage. 2019:184:266-78.

237. Wang $T$, et al. Regional homogeneity changes in patients with primary insomnia. Eur Radiol. 2016:26:1292-300.

238. Li X, et al. Increased interhemispheric resting-state functional connectivity in healthy participants with insomnia symptoms: a randomized clinical consort study. Medicine (Baltimore). 2017;96:e7037.

239. Li C, et al. Abnormal whole-brain functional connectivity in patients with primary insomnia. Neuropsychiatr Dis Treat. 2017;13:427-35.

240. Zhou $F$, et al. Temporal regularity of intrinsic cerebral activity in patients with chronic primary insomnia: a brain entropy study using resting-state fMRI. Brain Behav. 2016;6:e00529.

241. Zhao $\mathrm{L}$, et al. Cortical structural connectivity alterations in primary insomnia: insights from MRI-based morphometric correlation analysis. Biomed Res Int. 2015;2015:817595.

242. Chen MC, et al. Increased insula coactivation with salience networks in insom nia. Biol Psychol. 2014;97:1-8.

243. Dai XJ, et al. Altered intrinsic regional brain spontaneous activity and subjective sleep quality in patients with chronic primary insomnia: a resting-state fMRI study. Neuropsychiatr Dis Treat. 2014;10:2163-75.

244. Khazaie $\mathrm{H}$, et al. Functional reorganization in obstructive sleep apnoea and insomnia: a systematic review of the resting-state fMRI. Neurosci Biobehav Rev. 2017;77:219-31. 
Integrating sleep, neuroimaging, and computational approaches for...

AN. Goldstein-Piekarski et al.

204

245. Klumpp H, Hosseini B, Phan KL. Self-reported sleep quality modulates amygdala resting-state functional connectivity in anxiety and depression. Front Psychiatry. 2018;9:220.

246. Carlisi CO, et al. Sleep-amount differentially affects fear-processing neural circuitry in pediatric anxiety: a preliminary fMRI investigation. Cogn Affect Behav Neurosci. 2017;17:1098-113.
247. Clark $C P$, et al. Does amygdalar perfusion correlate with antidepressant response to partial sleep deprivation in major depression?. Psychiatry Res. 2006;146:43-51.

248. Soehner AM, et al. Cognitive control under stressful conditions in transitional age youth with bipolar disorder: diagnostic and sleep-related differences in fronto-limbic activation patterns. Bipolar Disord. 2018;20:238-47. 